\title{
Human SH2B1 mutations are associated with maladaptive behaviors and obesity
}

\author{
Michael E. Doche, ${ }^{1}$ Elena G. Bochukova, ${ }^{2}$ Hsiao-Wen Su, ${ }^{1}$ Laura R. Pearce, ${ }^{2}$ Julia M. Keogh, ${ }^{2}$ \\ Elana Henning, ${ }^{2}$ Joel M. Cline, ${ }^{1}$ Anne Dale, ${ }^{3}$ Tim Cheetham, ${ }^{4}$ Inês Barroso, $, 2,5$ \\ Lawrence S. Argetsinger, ${ }^{1}$ Stephen O’Rahilly, ${ }^{2}$ Liangyou Rui, ${ }^{1}$ \\ Christin Carter-Su, ${ }^{1}$ and I. Sadaf Farooqi ${ }^{2}$
}

\begin{abstract}
1Department of Molecular and Integrative Physiology, University of Michigan Medical School, Ann Arbor, Michigan, USA. 2University of Cambridge Metabolic Research Laboratories and NIHR Cambridge Biomedical Research Centre, Institute of Metabolic Science, Addenbrooke's Hospital, Cambridge, United Kingdom. ${ }^{3}$ Queen Elizabeth Hospital, Gateshead, United Kingdom. ${ }^{4}$ Royal Victoria Infirmary and Newcastle University, Newcastle-upon-Tyne, United Kingdom. ${ }^{5}$ Wellcome Trust Sanger Institute, Hinxton, United Kingdom.
\end{abstract}

\begin{abstract}
Src homology $2 \mathrm{~B}$ adapter protein 1 (SH2B1) modulates signaling by a variety of ligands that bind to receptor tyrosine kinases or JAK-associated cytokine receptors, including leptin, insulin, growth hormone (GH), and nerve growth factor (NGF). Targeted deletion of $S h 2 b 1$ in mice results in increased food intake, obesity, and insulin resistance, with an intermediate phenotype seen in heterozygous null mice on a high-fat diet. We identified SH2B1 loss-of-function mutations in a large cohort of patients with severe early-onset obesity. Mutation carriers exhibited hyperphagia, childhood-onset obesity, disproportionate insulin resistance, and reduced final height as adults. Unexpectedly, mutation carriers exhibited a spectrum of behavioral abnormalities that were not reported in controls, including social isolation and aggression. We conclude that $S H 2 B 1$ plays a critical role in the control of human food intake and body weight and is implicated in maladaptive human behavior.
\end{abstract}

\section{Introduction}

Leptin is a $16-\mathrm{kDa}$ circulating hormone that regulates energy homeostasis via hypothalamic neurons expressing the leptin receptor (LEPR) (1). Congenital deficiency of leptin and its receptor results in severe obesity in rodents and humans, implicating leptin-mediated signaling in the regulation of food intake, energy expenditure, carbohydrate metabolism, and neuroendocrine function $(2,3)$. Diet-induced obesity in rodents, and common forms of obesity in humans, are characterized by resistance to endogenous and exogenous leptin (4). Dissecting the intracellular signaling pathways and hypothalamic neural circuitry by which leptin exerts its effects is critical for the identification of potential therapeutic targets for obesity (5).

Leptin mediates effects on energy balance by binding to the long form of LEPR (LEPRb) and activating LEPRb-associated JAK2 (6). JAK2 phosphorylates multiple tyrosines in LEPRb (Tyr985/1107/1138), enabling recruitment of downstream effectors. JAK2 also autophosphorylates on Tyr813, allowing the binding of Src homology 2 (SH2) B adapter protein 1 (SH2B1), which enhances JAK2 activation and helps recruit IRS-1 and IRS-2 to the LEPRb/JAK2 complex $(7,8)$. This facilitates JAK2-mediated tyrosine phosphorylation of IRS- $1 / 2$ and subsequent activation of the PI3K pathway.

$\mathrm{SH} 2 \mathrm{~B} 1$ is a key endogenous positive regulator of leptin sensitivity; targeted deletion in mice results in impaired leptin signaling and severe obesity (8). Sh2b1-null mice are also insulin resistant and exhibit impaired insulin signaling (9). Multiple isoforms of SH2B1 are expressed in the brain; however, neuron-specific restoration of recombinant $S h 2 b 1 \beta$ alone is sufficient to reverse the

Authorship note: Michael E. Doche and Elena G. Bochukova, as well as Christin Carter-Su and I. Sadaf Farooqi, contributed equally to this work.

Conflict of interest: The authors have declared that no conflict of interest exists. Citation for this article: J Clin Invest. 2012;122(12):4732-4736. doi:10.1172/JCI62696. obesity observed in Sh2b1 knockout mice (10), which suggests that centrally expressed $\mathrm{SH} 2 \mathrm{~B} 1 \beta$ is critical to energy homeostasis.

To investigate the importance of SH2B1 in human energy homeostasis and insulin signaling, we sought to identify mutations in the $S H 2 B 1$ gene in a group of severely obese patients. We identified 300 patients with severe early-onset obesity from the Genetics of Obesity Study (GOOS) cohort (11) with a disproportionate degree of insulin resistance for their obesity, as defined by the presence of acanthosis nigricans, development of type 2 diabetes in early adolescence, and/or markedly elevated plasma insulin (top decile for age, gender, and BMI). Mutations in the genes causing the known monogenic obesity syndromes had been excluded in these patients, as had deletions at $16 \mathrm{p} 11.2$ by multiplex ligation-dependent probe amplification (MLPA) (12).

\section{Results and Discussion}

We identified 5 probands of mixed European descent with heterozygous mutations in SH2B1 (Figure 1A): a frameshift mutation, F344LfsX20, which leads to a truncated protein product, and 3 missense mutations, $\mathrm{P} 90 \mathrm{H}$ (2 patients), T175N, and P322S; all were absent from 500 control subjects $(P<0.001)$. Probands were apparently unrelated over 3 generations, as assessed by medical history. All mutations were inherited from overweight/obese parents, and carriers were hyperphagic and had reduced final height as adults (Table 1). Mutation carriers were hyperinsulinemic (fasting plasma insulin $>60 \mathrm{pmol} / \mathrm{l}$ ) and euglycemic; liver function tests and lipid profiles were within the normal range (data not shown). Unexpectedly, we found that mutation carriers were reported to have delayed speech and language development and aggressive behavior by healthcare professionals and by family members (Table 1). However, these individuals did not consent to further behavioral testing, so the precise nature and severity of these phenotypes could not be determined. None of the controls were reported to have behavioral abnormalities by healthcare professionals. 
A

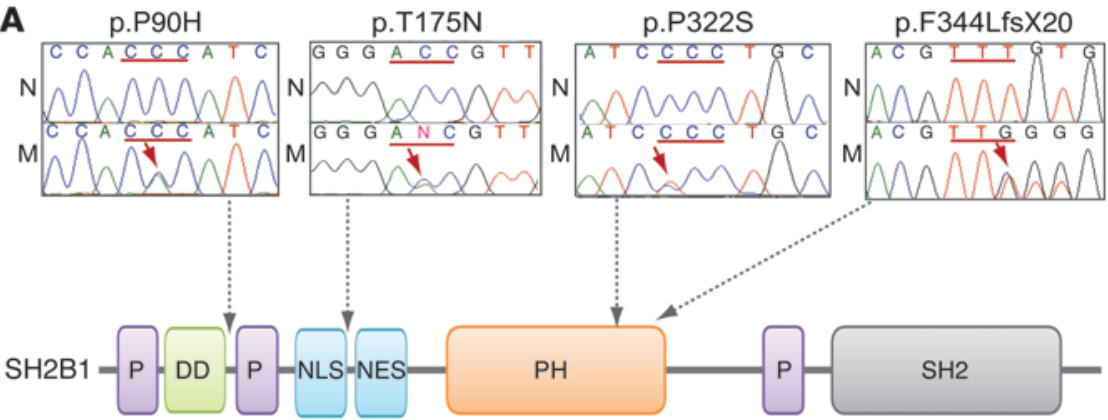

$\mathbf{B}$

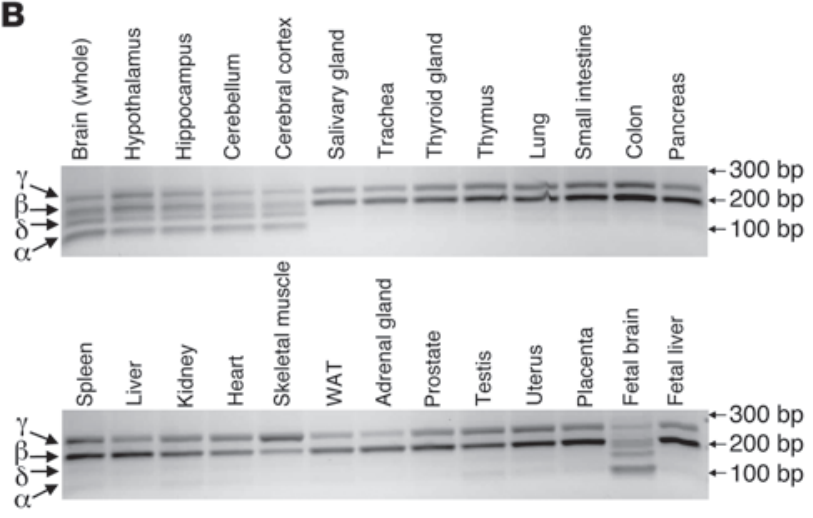

We next sought to assess the molecular and cellular basis for the phenotypes associated with these human mutations. SH2B1 is a member of a family of SH2 domain-containing adaptor molecules - SH2B1 (also known as PSM), SH2B2 (also known as APS), and $\mathrm{SH} 2 \mathrm{~B} 3$ (also known as Lnk) - that bind to activated receptor tyrosine kinases, including insulin receptor and TrkA, the receptor for nerve growth factor (NGF) (13). SH2B1 can also bind to activated JAK2 (14), a cytokine receptor-associated tyrosine kinase that is activated after binding of cytokine receptor ligands, such as leptin and growth hormone (GH). SH2B1 has an aminoterminal dimer-

\section{Figure 1}

Identification of $S H 2 B 1$ mutations. (A) Human SH2B1 protein (NP_001139268). P, proline-rich region; DD, dimerization domain; NLS, nuclear localization sequence; NES, nuclear export sequence; $\mathrm{PH}$, pleckstrin homology domain. SH2B1 mutations are shown, with nucleotide changes marked on the chromatograms (arrows). N, normal allele; $\mathrm{M}$, mutated allele. (B) Expression of the 4 SH2B1 isoforms $(\alpha, \beta, \gamma$, and $\delta$ ), studied by qualitative RT-PCR in a panel of human tissues.

ization domain, nuclear localization and export sequences, a central pleckstrin homology domain, and a carboxyterminal SH2 domain (Figure 1A). The SH2B1 transcript undergoes alternative splicing at the $3^{\prime}$ end, giving rise to 4 protein products (SH2B $1 \alpha$,

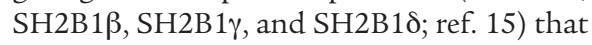
share their amino termini, nuclear localization sequence, nuclear export sequence, pleckstrin homology domain, and SH2 domain, but differ at their carboxyl termini. We investigated expression of the 4 SH2B1 isoforms in human tissues by RT-PCR (Figure $1 \mathrm{~B}$ ). As the $\beta$ isoform is the predominant form in the hypothalamus, we introduced the mutations into $\mathrm{SH} 2 \mathrm{~B} 1 \beta$ and examined their effect on SH2B1 $\beta$ expression, subcellular localization, and ability to enhance NGF-induced neuronal differentiation, cycling through the nucleus, GH-induced macrophage motility, JAK2 activation, leptin signaling, and insulin signaling.

Previous studies implicated SH2B1 in neuronal differentiation induced by NGF or by glial cell line-derived neurotrophic factor $(16-18)$. To test the mutations' effect on the ability of SH2B1 $\beta$ to enhance neuronal differentiation, we transiently expressed GFP-

Table 1

Phenotypic characteristics of SH2B1 mutation carriers

\begin{tabular}{|c|c|c|c|c|c|c|c|c|}
\hline & Mutation & $\begin{array}{l}\text { Age } \\
\text { (yr) }\end{array}$ & $\begin{array}{l}\text { Most recent } \\
\text { BMI }\left(\mathrm{kg} / \mathrm{m}^{2}\right)\end{array}$ & $\begin{array}{c}\text { BMI } \\
\text { (SD score) }\end{array}$ & $M / F$ & $\begin{array}{l}\text { Height } \\
\text { (SD score) }\end{array}$ & Genotype & Neurobehavioral phenotypes \\
\hline Proband & $\mathrm{P9OH}$ & 27 & 45 & - & M & 1.5 & NM & Social isolation, aggression \\
\hline Proband & T175N & 4 & 29 & 5.1 & $\mathrm{~F}$ & 2.8 & NM & Speech and language delay, aggression \\
\hline Mother & T175N & 31 & 30 & - & $\mathrm{F}$ & 0.4 & NM & Speech and language delay \\
\hline Father & T175N & 30 & 29 & - & $M$ & 1.1 & NN & Nil \\
\hline Sister & T175N & 7 & 21 & 2.2 & $\mathrm{~F}$ & -0.8 & NM & Speech and language delay \\
\hline Proband & P322S & 14 & 32 & 2.9 & M & -0.8 & NM & Social isolation, aggression \\
\hline Mother & P322S & 54 & 27 & - & $\mathrm{F}$ & 0.2 & NM & Nil \\
\hline Father & P322S & 55 & 22 & - & M & 0.9 & NN & Nil \\
\hline Brother & P322S & 18 & 32 & - & M & 0.8 & NM & Social isolation \\
\hline Proband & F344LfsX20 & 13 & 37 & 3.5 & $\mathrm{~F}$ & 0.8 & NM & Social isolation, aggression \\
\hline Mother & F344LfsX20 & 48 & 48 & - & $\mathrm{F}$ & -2.7 & NM & Social isolation \\
\hline Father & F344LfsX20 & 55 & 26 & - & M & 1.3 & NN & Nil \\
\hline Sister & F344LfsX20 & 21 & - & - & $\mathrm{F}$ & - & NN & Nil \\
\hline
\end{tabular}

Probands were identified by genetic screening; other individuals are family members of probands as indicated. Data were only included where reports from healthcare professionals and family members were available. -, data missing (in some cases, family members were deceased or contact information was not available); $\mathrm{N}$, normal allele; $\mathrm{M}$, mutant allele. 
A

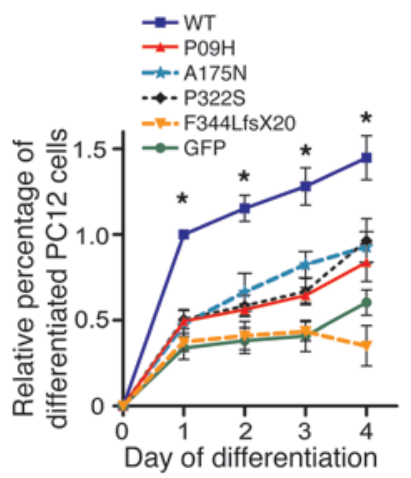

B
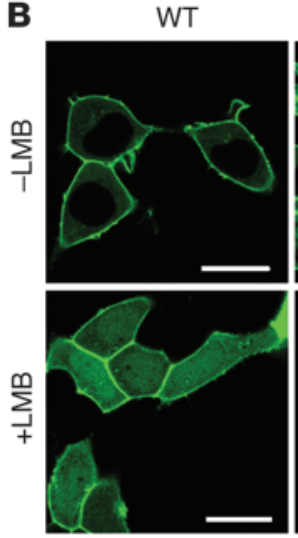

$\mathrm{P9OH}$
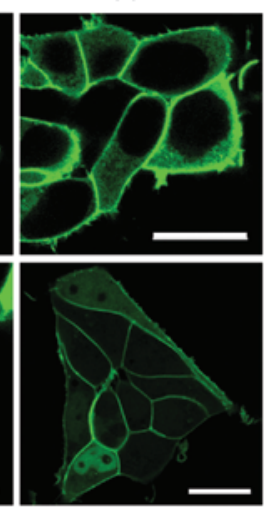

A175N
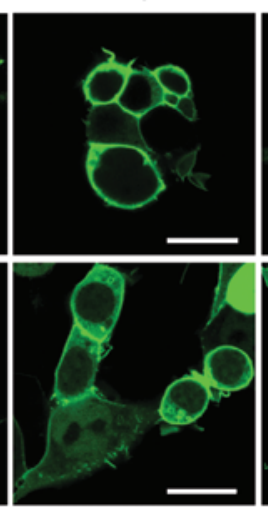

P322S
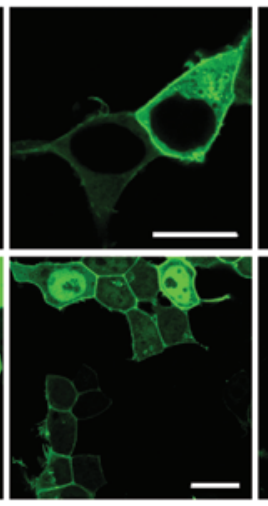

F344LfsX20
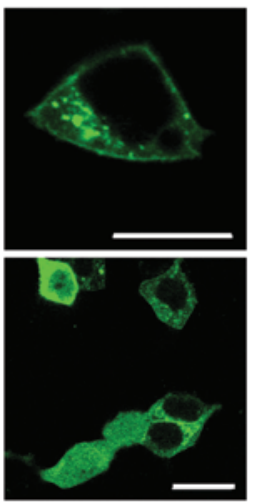

C

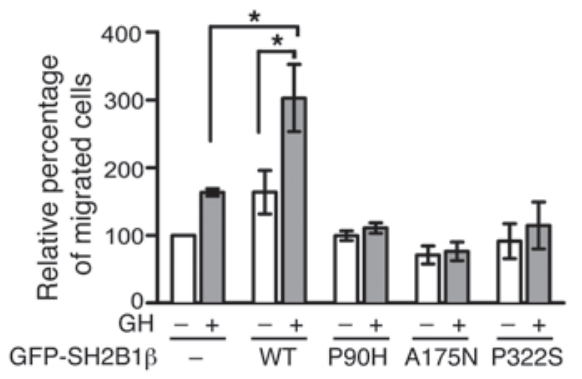

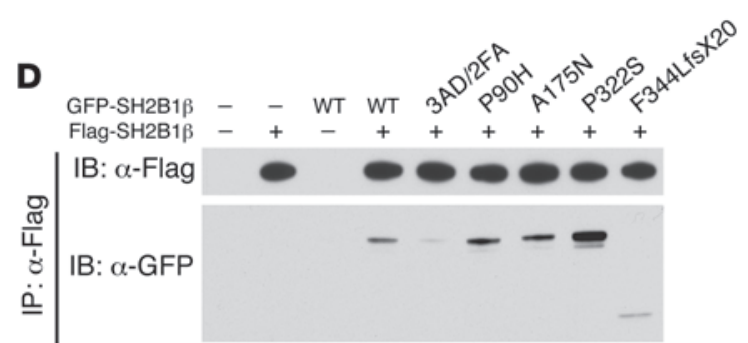

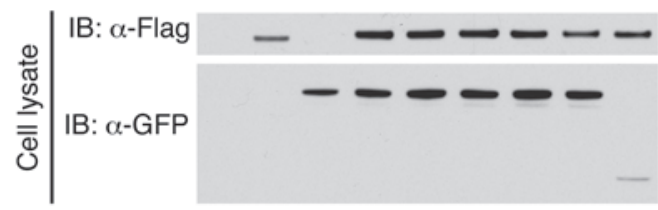

\section{Figure 2}

Functional characterization of SH2B1 mutations. (A) PC12 cells transiently expressing GFP-tagged WT or mutant SH2B1 $\beta$ were treated with NGF to induce differentiation. Percent differentiated values were normalized against WT at day 1 of differentiation (means \pm SEM). All mutants impaired the rate of NGF-induced neuronal differentiation compared with WT. ${ }^{*} P<0.0001$, 1-way ANOVA with Dunnett's post-test. (B) $293 T$ cells transiently expressing GFP-tagged WT or mutant SH2B1 $\beta$ were treated with or without leptomycin B (LMB) and imaged using confocal microscopy. Scale bars: $10 \mu \mathrm{m}$. All SH2B1 $\beta$ mutant proteins exhibited an impaired ability to accumulate in the nucleus (see Supplemental Figure 1). (C) RAW264.7 macrophages transiently expressing GFP-tagged WT or mutant SH2B1 $\beta$ were added to the upper chamber, and GH (500 $\mathrm{ng} / \mathrm{ml})$ to the lower chamber, of a Transwell plate. Average values for migrated cells were normalized to unstimulated control values (means \pm SEM). All SH2B1 $\beta$ mutants inhibited $\mathrm{GH}$-induced cell migration. ${ }^{*} P<0.05$. (D) Flag-tagged SH2B1 $\beta$ was immunoprecipitated from HEK293 cells coexpressing Flagtagged WT SH2B1 $\beta$ and GFP-tagged SH2B1 $\beta$ mutants (including the dimerization mutant 3AD/2FA). Immunoprecipitated proteins and proteins in cell lysates were immunoblotted using anti-Flag and anti-GFP antibodies. Binding of the $\mathrm{P} 90 \mathrm{H}$ and $\mathrm{A} 175 \mathrm{~N}$ mutants was similar to that seen with WT; however, the P322S mutation enhanced dimerization. Note that for the $\mathrm{A} 175 \mathrm{~N}$ mutant, all experiments were performed using rat $\mathrm{SH} 2 \mathrm{~B} 1 \mathrm{\beta}$ (NP_001041645), in which Thr175 is Ala175.

tagged WT or mutant SH2B1 $\beta$ in PC12 cells, treated the cells with NGF to induce neuronal differentiation, and determined the percentage of $\mathrm{GFP}^{+}$cells that were differentiated (neurite outgrowths $>2$ times the length of the cell body). The truncation mutation failed to enhance NGF-induced neuronal differentiation, and the $\mathrm{P} 90 \mathrm{H}, \mathrm{A} 175 \mathrm{~N}$, and $\mathrm{P} 322 \mathrm{~S}$ mutations significantly impaired the ability of SH2B $1 \beta$ to enhance neuronal differentiation compared with WT (Figure 2A).

Nuclear shuttling of SH2B $1 \beta$ appears to be required for its stimulatory effect on neuronal differentiation (19). To test whether the mutations impair the ability of SH2B $1 \beta$ to translocate to the nucleus, we treated $293 \mathrm{~T}$ cells expressing GFP-tagged forms of SH2B1 $\beta$ with an inhibitor of nuclear export, leptomycin B, for 8 hours. While confocal microscopy revealed that approximately $95 \%$ of the cells expressing GFP-tagged WT SH2B1 $\beta$ showed a nuclear/cytoplasmic GFP fluorescence ratio of $\geq 1$, cycling of the mutants into the nucleus was significantly impaired (Figure $2 \mathrm{~B}$ and Supplemental Figure 1; supplemental material available online with this article; doi:10.1172/JCI62696DS1).

SH2B1 has been implicated in GH regulation of the actin cytoskeleton (20). We therefore examined the ability of WT and mutant forms of SH2B1 $\beta$ to enhance GH-induced motility of cultured RAW264.7 macrophages. In contrast to GFP-tagged WT SH2B1 $\beta$, which stimulated both basal and GH-induced motility, the point mutants inhibited GH-induced motility (Figure 2C).

SH2B $1 \beta$ mutants $\mathrm{P} 90 \mathrm{H}, \mathrm{A} 175 \mathrm{~N}$, and $\mathrm{P} 322 \mathrm{~S}$ were expressed at the appropriate size and intensities and retained their ability to activate JAK2 (Figure 2D and data not shown). P90H, A175N, and P322S also exhibited a steady-state subcellular distribution similar to that of WT SH2B1 $\beta$ (Figure 3). In contrast, the F344LfsX20 mutant exhibited significantly reduced expression and was unable to activate JAK2 (Figure 2D and data not shown), presumably due to the fact that it lacks the $\mathrm{SH} 2$ domain previously shown to be required for JAK2 activation (21). SH2B1ß F344LfsX20 was also 
WT
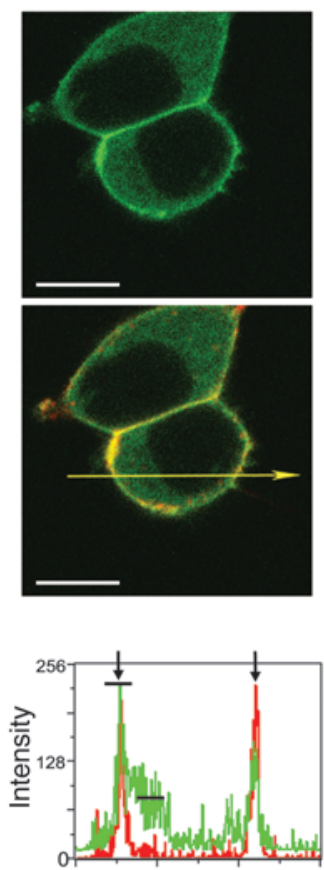

Relative position
$\mathrm{P} 90 \mathrm{H}$
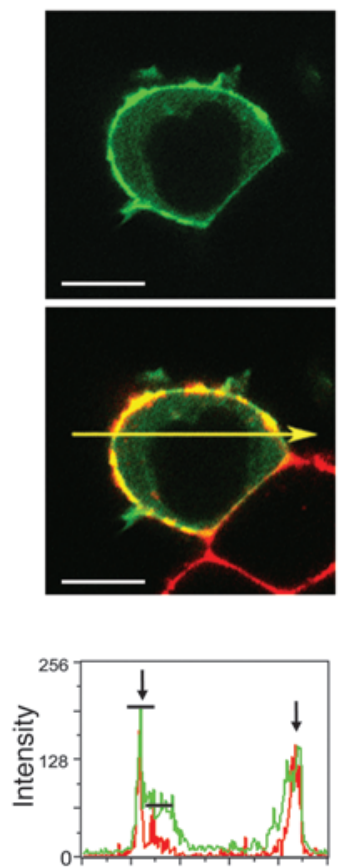

Relative position
A175N
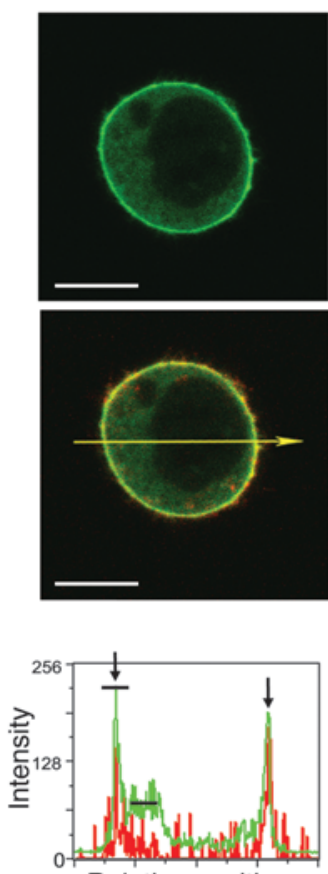

Relative position
P322S
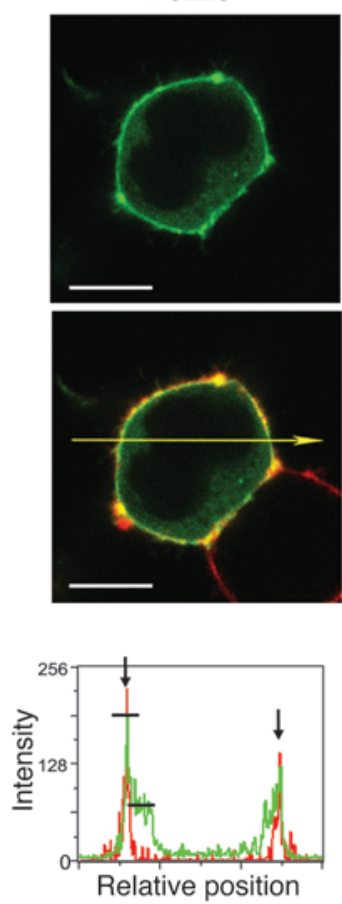

F344LfsX20
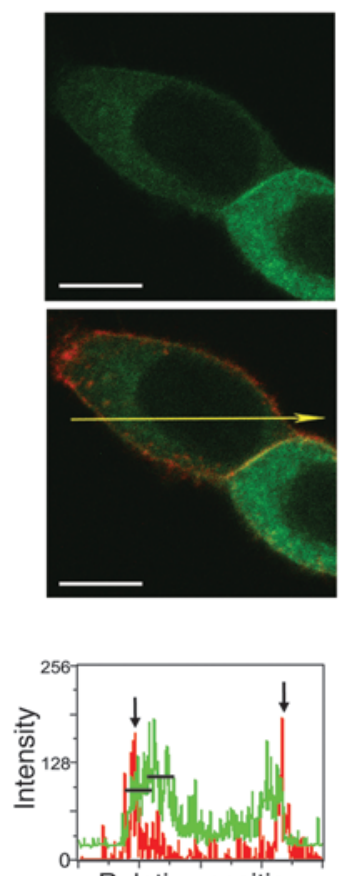

Relative position

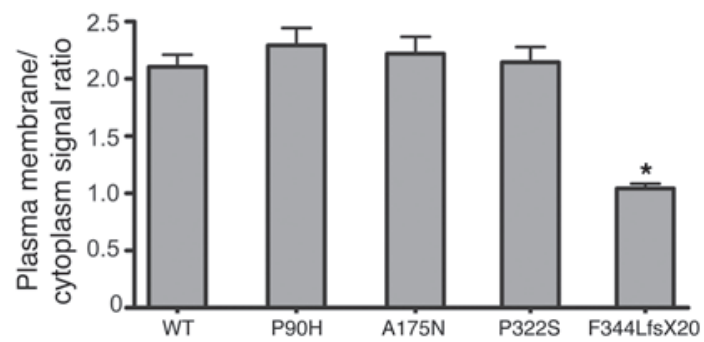

Figure 3

Subcellular distribution of SH2B1 $\beta$ WT and mutant proteins. Live 293T cells transiently expressing GFP-tagged WT, P90H, A175N, P322S, or F344LfsX20 SH2B1 $\beta$ were stained with the plasma membrane marker wheat germ agglutinin Alexa Fluor 594 (red) and imaged using confocal fluorescence microscopy. Shown are GFP fluorescence alone (green; top) and overlay of GFP and plasma membrane marker (bottom). Scale bars: $10 \mu \mathrm{m}$. Below, green and red signal intensity along the yellow arrows was determined using MetaVue Linescan. Arrows (red signal intensity peak) indicate position of the plasma membrane on the linescan. Horizontal lines on the linescan graphs denote the plasma membrane and cytoplasm values used to determine the plasma membrane/cytoplasm green signal intensity ratios (means $\pm \mathrm{SEM}$ ). The SH2B1 $\beta$ truncation mutant localized to the PM to a lesser extent than did SH2B1 $\beta$ WT. ${ }^{\star} P<0.0001$ vs. WT.

present at lower levels in the plasma membrane relative to the cytoplasm in both 293T and PC12 cells and was found in aggregates in the cytoplasm (Figure 3 and data not shown). We assessed the ability of the SH2B1 $\beta$ mutants to enhance leptin signaling by examining their ability to stimulate leptin-dependent tyrosyl phosphorylation of IRS2. Consistent with the findings on JAK2 activation, the SH2B1 $\beta$ point mutants were as effective as WT SH2B $1 \beta$ in stimulating leptin-dependent tyrosine phosphorylation of IRS2; similar results were seen for insulin-stimulated tyrosine phosphorylation of IRS2 (data not shown). As these latter assays rely on the overexpression of both SH2B1 $\beta$ and IRS2, it is possible that any subtle effects of the SH2B1 mutants were masked.

We also postulated that some of these heterozygous mutations may affect the ability of SH2B1 to dimerize. GFP-tagged WT SH2B1 $\beta$ coimmunoprecipitated with Flag-tagged WT SH2B1 $\beta$, while the mutant $3 \mathrm{AD} / 2 \mathrm{FA}$ (in which the phenylalanine zipper that is required for dimerization is mutated) exhibited greatly diminished coimmunoprecipitation (Figure 2D). Binding of the GFP-tagged P90H and A175N mutants to Flag-tagged WT SH2B1 $\beta$ was similar to that seen with GFP-tagged WT; however, the P322S mutation enhanced dimerization.

We have demonstrated that loss-of-function mutations in SH2B1 were associated with severe early-onset obesity, insulin resistance, and reduced final height. All the mutations were associated with loss of function in assays of GH/NGF-mediated signaling. Intriguingly, apart from the frameshift mutation, the other mutants did not impair leptin signaling. Although this discordance may reflect the differing sensitivities of the assays used, it is plausible that some of the effects of SH2B1 on energy homeostasis may be mediated by leptin-independent pathways.

Unexpectedly, we observed that mutations were associated with a range of behavioral abnormalities, including a tendency for social 
isolation and high levels of aggression, as reported by healthcare professionals and family members. We recognize that the absence of formal psychiatric testing and disease classification in these individuals and in the controls is a limitation of our study. However, we note that these phenotypes were not seen in association with the other genetic obesity syndromes we have characterized to date $(11,12)$, although the socioeconomic status of mutation carriers was comparable. As we observed impaired NGF-induced neuronal differentiation in vitro, a similar response to other ligands, such as centrally expressed neurotrophins, could contribute to these features (22). The maladaptive behaviors reported in mutation carriers compared with controls were not reported in previous studies in mice. Our studies imply an unexpected role for $\mathrm{SH} 2 \mathrm{~B} 1$ in human behavior.

\section{Methods}

SH2B1 mutation analysis. All 300 patients from the GOOS study that fit the criteria for obesity and severe insulin resistance were screened for mutations in the SH2B1 gene. An a priori power calculation was not conducted; given the rarity of these phenotypes, all eligible patients available to us were studied. Primers were designed to cover the coding sequence (NM_015503) and splice junctions of $S H 2 B 1$. Mutation screening was undertaken by PCR, followed by direct sequencing using BigDye terminator chemistry (Applied Biosystems) and analysis on an ABI 3730 automated sequencer (Applied Biosystems). 500 controls were also sequenced using the same methods.

SH2B1 gene expression in human tissues. A human tissue cDNA library was prepared using $1 \mu \mathrm{l}$ RNA (Clontech), which was reverse transcribed to cDNA using a RetroScript kit (Ambion). $1 \mu \mathrm{l}$ cDNA was used as a template in an RT-PCR reaction (forward primer, $5^{\prime}$-CAGCTATGTCCCATCCTCCCAGCGA-3'; reverse primer, 5'-CAGGCTGTGGGGGATCTGTCCATGAAG-3'). The 4 SH2B1 isoforms were amplified simultaneously, generating 4 PCR products of distinct size (SH2B1 $\alpha, 102$ bp; SH2B1 $\beta, 201$ bp; SH2B1 $\gamma, 254$ bp; SH2B18, 154 bp).

Statistics. See Supplemental Methods for details. 2-sided Fisher's exact test was used to compare prevalence of mutations in cases versus controls. A $P$ value less than 0.05 was considered significant.
Study approval. All studies were approved by the Anglia and Oxford multiregional ethics committee. Each subject, or parent (children $<16$ years), provided written informed consent (oral consent was obtained from the minors themselves). Clinical studies were conducted in accordance with the principles of the Declaration of Helsinki.

\section{Acknowledgments}

We are indebted to the patients and their families for their participation and to the physicians involved in the GOOS study. We thank S. Saeed, A. Thompson, H. Cha, and A. Ronen. This work was supported by the Wellcome Trust $(082390 / Z / 07 / Z$ and 077016/Z/05/Z), by Medical Research Council and NIHR Cambridge Biomedical Research Centre (to I.S. Farooqi, I. Barroso, and S. O'Rahilly); and by NIH grants RO1-DK54222 (to C. Carter-Su), RO1-DK065122 (to L. Rui) and RO1-DK073601 (to L. Rui) and a predoctoral fellowship from the Systems and Integrative Biology Training Grant NIH-T32-GM008322 (to M.E. Doche). Confocal microscopy was performed using the Morphology and Image Analysis Core of the Michigan Diabetes Research and Training Center (NIH grant P60-DK20572). cDNA sequencing was supported by the University of Michigan Comprehensive Cancer Center (NIH grant P30-CA46592).

Received for publication January 3, 2012, and accepted in revised form September 18, 2012.

Address correspondence to: I. Sadaf Farooqi, University of Cambridge Metabolic Research Laboratories, Institute of Metabolic Science, Box 289, Addenbrooke's Hospital, Cambridge CB2 0QQ, United Kingdom. Phone: 44.1223.762634; Fax: 44. 1223.762657; E-mail: isf20@cam.ac.uk. Or to: Christin CarterSu, University of Michigan Medical School, 6811C Medical Science 2, 1137 Catherine Street, Ann Arbor, Michigan 481095622, USA. Phone: 734.763.2561; Fax: 734.647.9523; E-mail: cartersu@umich.edu.
1. Zhang Y, Proenca R, Maffei M, Barone M, Leopold L, Friedman JM. Positional cloning of the mouse obese gene and its human homologue. Nature. 1994;372(6505):425-432.

2. Farooqi IS, et al. Effects of recombinant leptin therapy in a child with congenital leptin deficiency. NEngl J Med. 1999;341(12):879-884.

3. Clement $\mathrm{K}$, et al. A mutation in the human leptin receptor gene causes obesity and pituitary dysfunction. Nature. 1998;392(6674):398-401.

4. Myers MG Jr, Leibel RL, Seeley RJ, Schwartz MW Obesity and leptin resistance: distinguishing cause from effect. Trends Endocrinol Metab. 2010; 21(11):643-651.

5. Chua S. SH2B1-the adaptor protein that could. Endocrinology. 2010;151(9):4100-4102.

6. Robertson SA, Leinninger GM, Myers MG. Molec ular and neural mediators of leptin action. Physiol Behav. 2008;94(5):637-642.

7. Kurzer JH, Argetsinger LS, Zhou YJ, Kouadio JL, O'Shea JJ, Carter-Su C. Tyrosine 813 is a site of JAK2 autophosphorylation critical for activation of JAK2 by SH2-B beta. Mol Cell Biol. 2004;24(10):4557-4570.

8. Ren D, Li M, Duan C, Rui L. Identification of $\mathrm{SH} 2-\mathrm{B}$ as a key regulator of leptin sensitivity, energy balance, and body weight in mice. Cell Metab. 2005; 2(2):95-104

9. Morris DL, Cho KW, Zhou Y, Rui L. SH2B1 enhances insulin sensitivity by both stimulating the insulin receptor and inhibiting tyrosine dephosphorylation of insulin receptor substrate proteins. Diabetes. 2009;58(9):2039-2047.

10. Ren D, Zhou Y, Morris D, Li M, Li Z, Rui L. Neuronal SH2B1 is essential for controlling energy and glucose homeostasis. J Clin Invest. 2007;117(2):397-406.

11. Farooqi IS, Keogh JM, Yeo GS, Lank EJ, Cheetham $\mathrm{T}$, O'Rahilly S. Clinical spectrum of obesity and mutations in the melanocortin 4 receptor gene. N Engl J Med. 2003;348(12):1085-1095

12. Bochukova EG, et al. Large, rare chromosomal deletions associated with severe early-onset obesity. Nature. 2010;463(7281):666-670.

13. Maures TJ, Kurzer JH, Carter-Su C. SH2B1 (SH2-B) and JAK2: a multifunctional adaptor protein and kinase made for each other. Trends Endocrinol Metab. 2007;18(1):38-45.

14. Rui L, Carter-Su C. Identification of SH2-bbeta as a potent cytoplasmic activator of the tyrosine kinase Janus kinase 2. Proc Natl Acad Sci U S A. 1999; 96(13):7172-7177.

15. Yousaf N, Deng Y, Kang Y, Riedel H. Four PSM/ $\mathrm{SH} 2-\mathrm{B}$ alternative splice variants and their differential roles in mitogenesis. J Biol Chem. 2001; 276(44):40940-40948.

16. Qian X, Riccio A, Zhang Y, Ginty DD. Identification and characterization of novel substrates of Trk receptors in developing neurons. Neuron. 1998;21(5):1017-1029.

17. Rui L, Herrington J, Carter-Su C. SH2-B, a membrane-associated adapter, is phosphorylated on multiple serines/threonines in response to nerve growth factor by kinases within the MEK/ERK cascade. J Biol Chem. 1999;274(37):26485-26492.

18. Zhang Y, et al. Interaction of SH2-Bbeta with RET is involved in signaling of GDNF-induced neurite outgrowth. J Cell Sci. 2006;119(pt 8):1666-1676.

19. Maures TJ, Chen L, Carter-Su C. Nucleocytoplasmic shuttling of the adapter protein SH2B1beta (SH2-Bbeta) is required for nerve growth factor (NGF)-dependent neurite outgrowth and enhancement of expression of a subset of NGF-responsive genes. Mol Endocrinol. 2009;23(7):1077-1091.

20. Diakonova M, Gunter DR, Herrington J, Carter-Su C. SH2-Bbeta is a Rac-binding protein that regulates cell motility. J Biol Chem. 2002;277(12):10669-10677.

21. Rui L, Mathews LS, Hotta K, Gustafson TA, Carter-Su C. Identification of SH2-Bbeta as a substrate of the tyrosine kinase JAK2 involved in growth hormone signaling. Mol Cell Biol. 1997;17(11):6633-6644.

22. Gray J, et al. Hyperphagia, severe obesity, impaired cognitive function, and hyperactivity associated with functional loss of one copy of the brain-derived neurotrophic factor (BDNF) gene. Diabetes. 2006; 55(12):3366-3371. 